\title{
Um Ambiente Virtual Aplicado ao Ensino e Pesquisa em Arquitetura e Organização de Computadores
}

\author{
Guilherme Esmeraldo', Edson Barbosa Lisboa² \\ ${ }^{1}$ Instituto Federal de Educação, Ciência e Tecnologia do Ceará (IFCE) - \\ Crato, CE - Brazil \\ ${ }^{2}$ Instituto Federal de Educação, Ciência e Tecnologia de Sergipe (IFS) - Aracaju, SE - \\ Brazil \\ guilhermealvaro@ifce.edu.br, edson.barbosa@ifs.edu.br
}

\begin{abstract}
Studies and research in architecture and computer organization has gained even more relevance due to the rapid technological evolution, which has made available a variety of increasingly complex computing platforms. The big challenge is to understand and master the peculiarities of new technologies. For this, the use of virtual tools have been increasingly stimulated. Thus the purpose of this work is to present an integrated environment for the design and simulation of computational platforms that can be easily configured and expandable, raising the level of abstraction and facilitating the understanding of the integration between hardware and software.
\end{abstract}

Resumo. O estudo e pesquisa em arquitetura e organização de computadores têm ganhado ainda mais relevância em função da rápida evolução tecnológica, que têm disponibilizado uma variedade de plataformas computacionais cada vez mais complexas. O grande desafio é entender e dominar as peculiaridades das novas tecnologias. Para isso, o uso de ferramentas virtuais têm sido cada vez mais estimulado. Assim o propósito desse trabalho é apresentar um ambiente integrado de concepção e simulação de plataformas computacionais que possam ser facilmente configuradas $e$ expansíveis, elevando o nível de abstração e facilitando o entendimento da integração entre hardware e software.

\section{Introdução}

O estudo e projeto integrado de hardware e software tem se tornado ainda mais relevante devido aos grandes avanços tecnológicos, que têm disseminado uma vasta quantidade de plataformas computacionais para atender diferentes requisitos tecnológicos, tais como computação paralela, sistemas embarcados, internet das coisas (IoT) e automação [Ecker, 2009] [Atzori, 2010]. Esse contexto implica em um grande desafio para o ensino e pesquisa em ambientes acadêmicos devido à complexidade inerente à alta escala de integração, o que dificulta a percepção de conceitos e funcionalidades presentes em arquiteturas e plataformas modernas.

Portanto, o uso de ambientes virtuais de simulação como abordagem de ensino e pesquisa tem sido fortemente recomendado, pois permitem simplificar e dinamizar a criação e a simulação de novos sistemas computacionais em cenários próximos aos reais. Assim, o objetivo desse trabalho é propor uma ferramenta de simulação para apoiar o projeto e a aprendizagem em arquitetura e organização de computadores. A ferramenta proposta, chamada de CompSim, suporta uma abordagem para projeto e 
estudo integrado dos conceitos relacionados aos componentes de hardware, suas funções e interações em si, bem como sua programação em baixo nível.

\section{Trabalhos Relacionados}

Os ambientes de simulação como o HADES (Hamburg Design System) [Hendrich, 2002] e o DEEDS (Digital Electronic Education Design Suite) [Donzellini; Ponta, 2013] possibilitam o estudo desde portas lógicas a sistemas microprocessados completos, em diferentes níveis de abstração. No entanto, a configuração e parametrização de componentes específicos não é algo tão simples, devido à complexidade do ambiente proposto. Abordagens como CPUSIm [Skrien, 2001] e SimuS [Silva; Borges, 2016] incluem interfaces gráficas que permitem a configuração e interação com os componentes de hardware e o ambiente de simulação, e focam essencialmente na exploração da programação em baixo nível. Esses simuladores, no entanto, por não incluírem suporte a alguns tipos de modelos de componentes ou ocultarem detalhes importantes para abstração da simulação, tornam-se ambientes de simulação distantes dos reais. Já os ambientes de simulação, como o VisiMips, são fortemente baseados no processo de animação da simulação em interface gráfica, vinculando a programação de baixo e médio nível com o comportamento dinâmico das unidades funcionais do processador [Kabir; Bari; Haque, 2011]. No entanto, esse simulador só dá suporte a um único tipo de processador. Por outro lado, simuladores como o MPSoCBench [Duenha; Azevedo, 2016], que apresentam componentes de hardware modelados seguindo todas as características de componentes reais, se tornam muito complexos de configurar e interagir.

Este trabalho apresenta o CompSim, um simulador gráfico que suporta o projeto de diferentes modelos de componentes de hardware, os quais podem ser facilmente configurados e simulados, além de trazer informações detalhadas e estatísticas de simulação sobre eles. A seção a seguir traz mais detalhes do simulador proposto.

\section{O Ambiente Virtual CompSim}

O simulador aqui proposto possui um ambiente gráfico integrado ${ }^{1}$ com diferentes recursos para apoio ao projeto e estudos de sistemas computacionais. Entre os recursos, pode-se citar: 1) Editor de código - com ele é possível codificar uma nova aplicação em baixo nível para execução no simulador; 2) CPU - exibe, em tempo de execução, os estados assumidos pelos registradores de um determinado processador; 3) CACHE MEMORY - exibe os estados das linhas de memória cache; 4) MAIN MEMORY exibe os estados dos diferentes endereços da memória principal; 5) DEVICES - exibe informações de comunicações de entrada e saída com os periféricos durante uma simulação; 6) SIMULATION - esse componente possibilita configurar e controlar as simulações; 7) LOG - neste componente são exibidas informações dos eventos e estatísticas de simulação gerados por cada um dos componentes de hardware; e 8) Assembler - valida um novo programa de máquina (análises léxica e sintática), e gera código binário executável.

O simulador CompSim foi codificado - componentes de simulação de hardware e interface gráfica - com a linguagem de programação Python 3.5 e compilado com 0 framework Nuitka² para as plataformas MS Windows e GNU/Linux (32 e 64 bits).

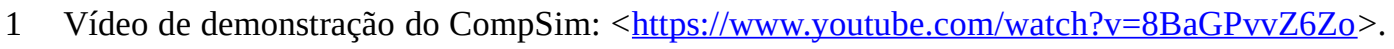

2 Nuitka. Nuitka Home. Disponível em: <http://www.nuitka.net/>. Acesso em: 07 mar. 2017. 


\section{Estudo de Caso: Processador Cariri}

Para avaliação da abordagem de projeto utilizando o simulador proposto, criou-se um modelo de simulação de um novo processador simples de 16 bits, denominado Cariri, o qual possui um novo conjunto de instruções (ISA - Instruction Set Architecture). Suas principais unidades funcionais e o caminho de dados (datapath) podem ser vistos na Figura 1.

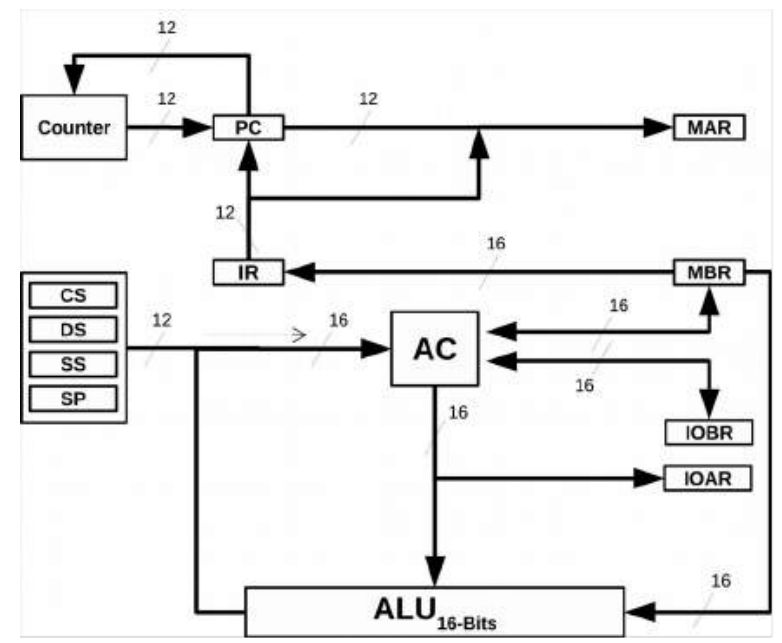

Figura 1. Processador Cariri: Unidades Funcionais e Datapath.

Entre as principais características desse processador, destacam-se: uma unidade lógica e aritmética (ULA), espaço de endereçamento diferenciado para entrada/saída e acesso à memória principal; suporte a diferentes modos de endereçamento; um banco de registradores para suportar 16 instruções de baixo nível; e suporte a operandos inteiros de 16-bits com sinalização (signed int) e a caracteres (bytes).

A plataforma computacional, criada para suportar a execução do novo modelo de processador, inclui memórias RAM e Cache, dispositivos de Entrada/Saída e um barramento. Para a simulação, utilizou-se uma aplicação Assembly, criada utilizando a ISA do processador Cariri, para cálculo iterativo do fatorial do número inteiro 7 (o resultado do cálculo do fatorial desse número está dentro do intervalo numérico que pode ser representado por um inteiro de 16-bits com sinal).

A Tabela 1 apresenta as estatísticas geradas pelos modelos de componentes da plataforma do estudo de caso, ao final da execução da aplicação de fatorial.

Tabela 1 - Estatísticas dos componentes de hardware para a aplicação de fatorial.

\begin{tabular}{|c|c|c|c|}
\hline \multicolumn{4}{|c|}{ Componente } \\
\hline CPU & Memória Cache & Memória Principal & Barramento \\
\hline 1. Instruções: 905 & 1.Acessos: 1707 & 1.Acessos: 3311 & 1. Transações: 1707 \\
\hline \multirow{5}{*}{$\begin{array}{l}\text { Arit./Lógicas: } 252 \\
\text { Transfer. Dados: } 501 \\
\text { Controle: } 151 \\
\text { Sistema: } 1\end{array}$} & $\begin{array}{l}\text { Leituras.:1500 } \\
\text { Escritas: } 207\end{array}$ & $\begin{array}{l}\text { Leituras: } 3104 \\
\text { Escritas: } 207\end{array}$ & $\begin{array}{l}\text { Leituras:1500 } \\
\text { Escritas: } 207\end{array}$ \\
\hline & 2. Cache Hits: 1319 & 2. Blocos acessados: 388 & 2. Trans. Memoria:1707 \\
\hline & $\begin{array}{l}\text { Hits (leitura): } 1189 \\
\text { Hits (escrita): } 130\end{array}$ & $\begin{array}{l}\text { Leituras: } 388 \\
\text { Escritas: } 0\end{array}$ & $\begin{array}{l}\text { Leituras: } 1500 \\
\text { Escritas: } 207\end{array}$ \\
\hline & 3. Cache Misses: 388 & 3.Bytes transf.: 6622 & 3. Transações de E/S: 0 \\
\hline & $\begin{array}{l}\text { Misses (leitura): } 311 \\
\text { Misses (escrita): } 77\end{array}$ & $\begin{array}{l}\text { Leituras: } 6208 \\
\text { Escritas: } 414\end{array}$ & $\begin{array}{l}\text { Leituras: } 0 \\
\text { Escritas: } 0\end{array}$ \\
\hline
\end{tabular}




\section{Conclusão e Trabalhos Futuros}

O uso de simuladores é uma prática recomendada no meio acadêmico, tanto para o aprendizado quanto para o projeto de novos sistemas computacionais. Acredita-se que o simulador proposto neste artigo permitirá otimizar a aprendizagem em arquitetura e organização de computadores, através de uma abordagem para estudo integrado dos conceitos relacionados aos componentes de hardware, bem como o projeto de novos sistemas computacionais. Trabalhos futuros incluem suportar diferentes arquiteturas de processadores, plataformas de hardware especificadas em linguagem de descrição de hardware (HDL) e possibilitar a interação com outros ambientes por meio de cossimulação.

\section{Referências}

Atzori, L.; Iera, A.; Morabito, G. (2010) The Internet of Things: A survey. Elsevier.

Donzellini, G.; Ponta, D. (2013) From Gates to FPGA: Learning Digital Design with Deeds. In: Proceedings of the Third Interdisciplinary Engineering Design Education Conference - IEDEC. pp.41-48.

Duenha, L.; Azevedo, R. Utilização dos Simuladores do MPSoCBench para o Ensino e Aprendizagem de Arquitetura de Computadores. In: International Journal of Computer Architecture Education (IJCAE), V. 5, n. 1, 2016. pp 26-31.

Ecker, W.; Müller. W., Dömer , R. (2009) Hardware-dependent Software Principles and Practice. Springer.

Hendrich, N. (2002) From CMOS-Gates to Computer Architecture: Lessons Learned from Five Years of Java-Applets. In: Proceedings of the 4th European Workshop on Microelectronics Education, EWME. Pp 23-24.

Kabir, M. T.; Bari, M. T.; Haque, A. L. (2011) VisiMips: Visual Simulator of MIPS32 Pipelined Processor. In: International Conference on Computer Science \& Education (ICCSE). p. 788-793.

Silva, G. P; Borges, J. A. dos S. SimuS: Um Simulador para o Ensino de Arquitetura e Organização de Computadores. In: International Journal of Computer Architecture Education (IJCAE). V. 5, n. 1, 2016. pp 7-12.

Skrien, D. (2001). CPU Sim 3.1: A tool for simulating computer architectures for computer organization classes. In: Journal on Educational Resources in Computing (JERIC), 1(4), 46-59. 